\title{
AN AUTONOMOUS HARMONIC FILTERING STRATEGY FOR DISTRIBUTED ENERGY RESOURCES CONVERTERS IN MICROGRID
}

\author{
Tzung-Lin Lee*, Chia-Tse Lee** and Po-Tai Cheng** \\ * Dept. of Electrical Engineering, National Sun Yat-sen University, Kaohsiung, TAIWAN \\ ${ }^{* *}$ Center for Advanced Power Technologies, Dept. of Electrical Engr., National Tsing Hua University, Hsinchu, TAIWAN
}

\begin{abstract}
Distributed generation based on emerging energy technologies has become an attractive alternative to the conventional centralized power stations, and has drawn much interest due to the increasing demand of premium electric power. The Microgrid is a great example of integrating distributed energy resources through power electronics interface converters for grid-connected or islanding operation. This paper presents a cooperative harmonic filtering strategy for the interface converters of distributed energy sources to address harmonic issues in a low-voltage Microgrid system. A droop control method based on the reactive volt-ampere consumption of harmonics of each interface converter is designed and implemented, to accomplish the overall harmonic filtering workload can be evenly shared among converters without any communications. The operation principle is discussed in details. Computer simulations and laboratory test results are also presented to validate the distributed harmonic damping capability of the proposed strategy.
\end{abstract}

Keywords - Distributed generation systems, Microgrid, harmonic droop control, harmonic damping.

\section{INTRODUCTION}

The development of distributed energy resources (DERs) technologies, such as photovoltaics, wind power, fuel cells and micro turbines, have been making great strides. By integrating DERs through power electronics converters, the Microgrid $[1,2]$ is one of the key technologies of distributed power systems which deliver premium electric power with high quality and reliability. Based on frequency and voltage droop controls, fundamental real power and reactive power can be shared among various DER converters in a decentralized fashion without any communication systems [3-6].

As the generation capacity within the Microgrid is often limited, the harmonic current drawn by nonlinear loads could easily result in harmonic voltage distortion. The concentration of DER converters also induces harmonic resonances [7, 8]. Various control techniques for reducing output voltage distortion of the inverter have been presented, including installing passive filters and employing active damping control [9-11]. However, these methods are intended for a single converter only. Bandwidth-harmonic power droop has been proposed to share the harmonic power among multiple converters [12]. High frequency signals of small magnitude are injected by the converter and sensed back to facilitate its

Manuscript received October 6, 2009; revised November 16, 2009 Accepted by recommendation of the Associate Editors.E. H. WAtanabe and J. A. Pomilio. control. This method requires high PWM frequency with fine resolution, and thus is suitable for applications of lower $\mathrm{kVA}$ rating. Blaabjerg et al. propose an inverter which operates as an active inductor at a certain harmonic frequency to absorb the harmonic current produced by nonlinear loads [13]. This approach is suitable if converters are installed at the same location. Akagi et al. propose the voltage detection active filter, in which the active filter behaves as a harmonic conductance, to reduce voltage harmonics in the power system [8].

An autonomous harmonic filtering strategy is presented in this paper for the DER converters in the Microgrid system [14]. In addition to the real power-frequency $(P-f)$ droop and the reactive power-voltage $(Q-V)$ droop controls, the DER converters operate as an active harmonic conductance to absorb harmonic current, and a droop control between the active harmonic conductance $(G)$ and the harmonic reactive power $(H)$ is proposed to accomplish even sharing of harmonic filtering workload. The proposed $G-H$ droop can be integrated together with the $P-f$ droop and the $Q-V$ droop of the DER converters so they can share the real power, the reactive power and the harmonic reactive power in a truly autonomous fashion without any communications.

\section{OPERATION PRINCIPLES}

A simplified one-line diagram of a low-voltage Microgrid system is shown in Figure 1. Multiple DER converter units $\left(\mathrm{DCU}_{x}, x=1 \cdots N\right)$, implemented by voltage source inverters, are installed in various locations within the system. The control of each DCU consists of the droop controller and the voltage controller. The droop controller determines the fundamental voltage command and the harmonic conductance command of the inverter based on the $P-f, Q-V$ and $G-H$ droop characteristics. The fundamental voltage command and the harmonic conductance command are then passed to the voltage controller to establish the desired power output and harmonic voltage suppression characteristic. Operation principles are elaborated as follows.

\section{A. Droop controller}

The droop controller calculates the power output of each DCU, and then determines the fundamental voltage command and the harmonic conductance command. The real power, reactive power and harmonic var can be calculated as follows $[15,16]$,

$$
\begin{gathered}
{\left[\begin{array}{l}
p \\
q
\end{array}\right]=\left[\begin{array}{l}
P+\tilde{p} \\
Q+\tilde{q}
\end{array}\right]=\left[\begin{array}{cc}
v_{\alpha} & v_{\beta} \\
-v_{\beta} & v_{\alpha}
\end{array}\right] \cdot\left[\begin{array}{l}
i_{\alpha} \\
i_{\beta}
\end{array}\right]} \\
H=\sqrt{\left(\tilde{p}_{R M S}\right)^{2}+\left(\tilde{q}_{R M S}\right)^{2}}
\end{gathered}
$$




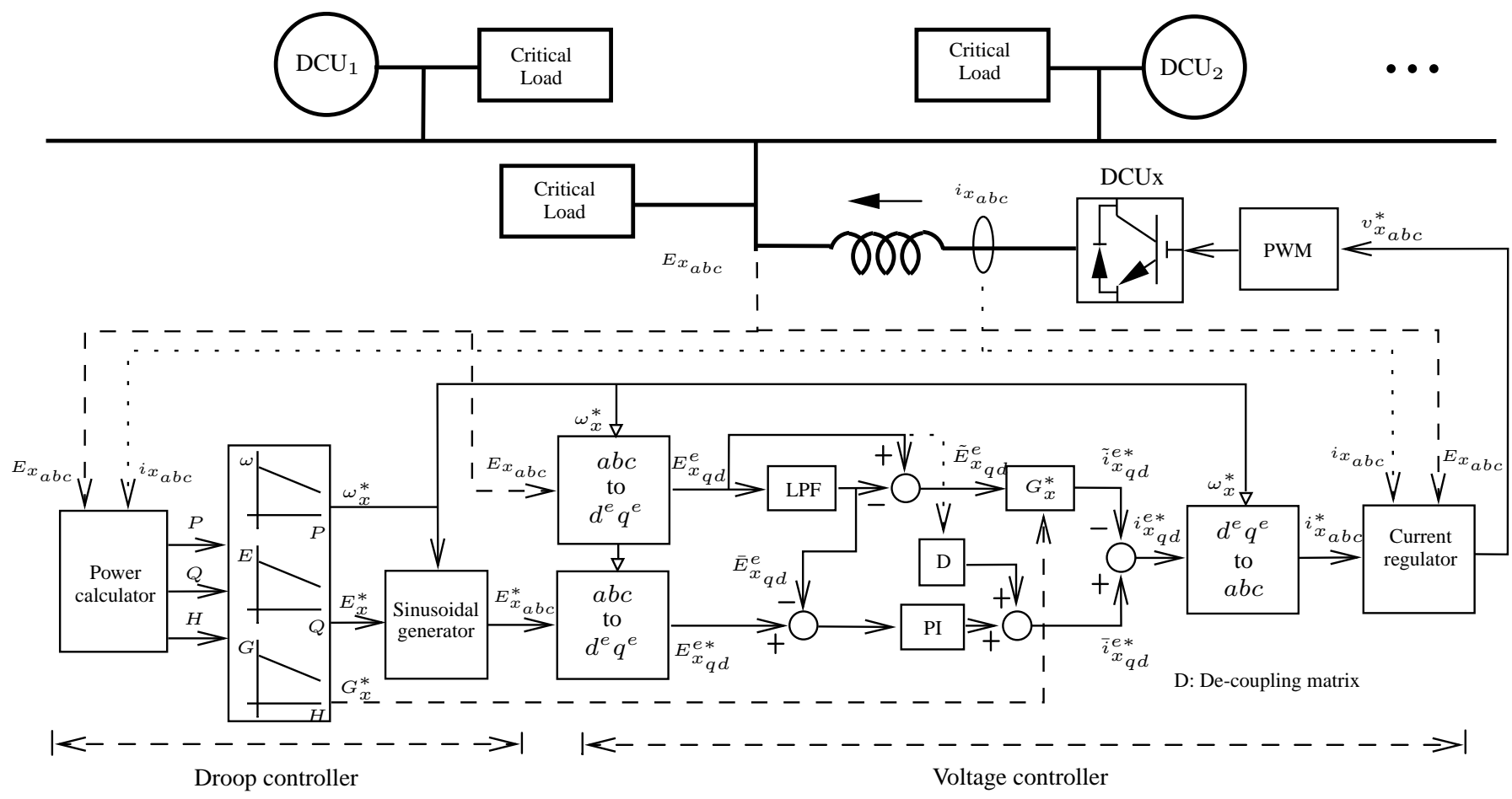

Fig. 1. Multiple distributed generation units in an islanding network and the control block diagram of the proposed harmonic filtering strategy.

where $p, q$ are the instantaneous real power and the instantaneous reactive power, respectively, $\tilde{p}_{R M S}$ and $\tilde{q}_{R M S}$ represent rms values of $\tilde{p}$ and $\tilde{q}$, respectively. The DC component $P$ and $Q$ represent the real power and the reactive power at the fundamental frequency, and $H$ is the harmonic reactive power.

The active harmonic conductance command $G_{x}^{*}$ is a control gain of DCUx for suppressing voltage harmonics. DCUx appears resistive at harmonic frequencies to absorb harmonics current, and to damp harmonics resonance. $G_{x}^{*}, \omega_{x}^{*}$, and $E_{x}^{*}$ of the DCUx are determined by the $G-H, Q-f$, and $P-V$ droop characteristics as follows,

$$
\begin{aligned}
G_{x}^{*} & =G_{0}-b_{x} \cdot\left(H_{0 x}-H_{x}\right) \\
\omega_{x}^{*} & =\omega_{0}-m_{x} \cdot\left(P_{0 x}-P_{x}\right) \\
E_{x}^{*} & =E_{0}-n_{x} \cdot\left(Q_{0 x}-Q_{x}\right)
\end{aligned}
$$

where

$b_{x}$ : harmonic droop coefficient; $m_{x}$ : frequency droop coefficient; $n_{x}$ : voltage droop coefficient; $G_{0}$ : rated conductance; $\omega_{0}$ : nominal frequency; $E_{0}$ : rated phase voltage magnitude; $H_{0 x}$ : harmonic var rated capacity; $Q_{0 x}$ : reactive power rated capacity; $P_{0 x}$ : real power rated capacity.

If the $P-f$ and the $Q-V$ droop characteristics of all DCUs are defined as

$$
\begin{aligned}
& m_{1} \cdot P_{01}=m_{2} \cdot P_{02}=\cdots=m_{x} \cdot P_{0 x}=\cdots=m_{N} \cdot P_{0 N} \\
& n_{1} \cdot Q_{01}=n_{2} \cdot Q_{02}=\cdots=n_{x} \cdot Q_{0 x}=\cdots=n_{N} \cdot Q_{0 N}
\end{aligned}
$$

then the real power and the reactive power can be shared in proportion to the rated capacity of each unit without any communications [3].

$$
\begin{aligned}
& P_{1}: P_{2}: \cdots \cdots: P_{x}: \cdots \cdots: P_{N} \\
& =P_{01}: P_{02}: \cdots \cdots: P_{0 x}: \cdots \cdots: P_{0 N} \\
& Q_{1}: Q_{2}: \cdots \cdots: Q_{x}: \cdots \cdots: Q_{N} \\
& =Q_{01}: Q_{02}: \cdots \cdots: Q_{0 x}: \cdots \cdots: Q_{0 N}
\end{aligned}
$$

Based on the $P-f$ droop and the $Q-V$ droop, the fundamental output voltage command $E_{x_{a b c}}^{*}$ can be determined.

The $G-H$ droop is defined in a similar fashion as follows,

$$
b_{1} \cdot H_{01}=b_{2} \cdot H_{02}=\cdots=b_{x} \cdot H_{0 x}=\cdots=b_{N} \cdot H_{0 N}
$$

The harmonic var consumption of the DCU primarily results from the fundamental voltage at the installation point and its harmonic current. Since the fundamental line voltages within the network is sufficiently close, the $G-H$ droop control allows even sharing of harmonic var among DCUs.

$$
\begin{aligned}
& H_{1}: H_{2}: \cdots \cdots: H_{x}: \cdots \cdots: H_{N} \\
& =H_{01}: H_{02}: \cdots \cdots \cdot H_{0 x}: \cdots \cdots: H_{0 N}
\end{aligned}
$$

The $G-H$ droop determines the harmonic conductance command $G_{x}^{*}$ for voltage harmonics suppression.

With the $P-f, Q-V$, and $G-H$ droop controllers, the DCUs can adjust the output fundamental frequency, the voltage magnitude and the harmonic conductance independently so the DCUs can share real power, reactive power, and harmonic var without any communications. 


\section{B. Voltage controller}

The voltage controller takes orders from the harmonic conductance command $G_{x}^{*}$ and the voltage command $E_{x_{a b c}}^{*}$ of the droop controller, and generates PWM switching signals of the DCU inverter. The three-phase voltage $E_{x_{a b c}}$ is measured and transformed into $E_{x_{q d}}^{e}$ in the synchronous reference frame. The fundamental voltage $\bar{E}_{x_{q d}}^{e}$ can be extracted by low-pass filters (LPF) from $E_{x_{q d}}^{e}$, and the harmonic voltage $\tilde{E}_{x_{q d}}^{e}$ can be obtained by subtracting $\bar{E}_{x_{q d}}^{e}$ from $E_{x_{q d}}^{e}$. The voltage harmonics $\tilde{E}_{x_{q d}}^{e}$ is multiplied by the harmonic conductance command $G_{x}^{*}$ to generate harmonic current commands $\tilde{i}_{x_{q d}}^{e *}$ of the inverter.

The fundamental current command $\bar{i}_{x_{q d}}^{e *}$ is generated by the closed-loop proportional-integral (PI) control between the fundamental voltage reference $E_{x_{q d}}^{e *}$ of the droop controller and the fundamental voltage $\bar{E}_{x_{q d}}^{e}$ extracted by LPF from $E_{x_{q d}}^{e}$. The decoupling between the $d^{e}$ and $q^{e}$ axes components is also included. The total current command $i_{x_{q d}}^{e *}$ consists of the fundamental current command $\bar{i}_{x_{q d}}^{e *}$ and the harmonic current command $\tilde{i}_{x_{q d}}^{e *}$ to facilitate the control of both the fundamental power and harmonics suppression.

The synchronous reference frame current command $i_{x_{q d}}^{e *}$ is then transformed back to the three-phase current command $i_{x_{a b c}}^{*}$. Based on $i_{x_{a b c}}^{*}$, the measured current $i_{x_{a b c}}$, and the measured voltage $E_{x_{a b c}}$, the current regulator calculates the voltage command $v_{x_{a b c}}^{*}$ as,

$$
v_{x_{a b c}}^{*}=\frac{L_{x}}{\Delta T}\left(i_{x_{a b c}}^{*}-i_{x_{a b c}}\right)+E_{x_{a b c}}
$$

where $L_{x}$ is the output inductor of the inverter, and $\Delta T$ is the sampling period of the digital controller. In order to track the current command with a high bandwidth, the spacevector PWM is employed to generate the corresponding gating signals so the inverter can synthesize the desired current.

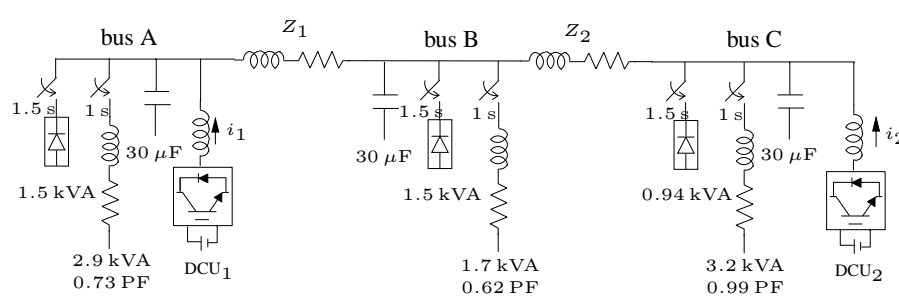

Fig. 2. Simulation circuit.

\section{TABLE I}

DCU parameters.

\begin{tabular}{|c|c|c|c|c|c|c|}
\hline & \multicolumn{2}{|c|}{$m(\mathrm{rad} / \mathrm{s} / \mathrm{W}) n(1 / \mathrm{A}) b\left(1 / \mathrm{V}^{2}\right.$} & \multicolumn{4}{|c|}{$\mathrm{P}_{0}(\mathrm{~kW}) \mathrm{Q}_{0}($ var $) \mathrm{H}_{0}(\mathrm{kvar}) G_{0}(1 / \Omega)$} \\
\hline $\mathrm{DCU}_{1}$ & $-10^{-5}$ & $-10^{-3}-10^{-2}$ & 5.0 & 0 & 2.0 & 0 \\
\hline $\mathrm{DCU}_{2}$ & $-10^{-5}$ & $-10^{-3}-10^{-2}$ & 5.0 & 0 & 2.0 & 0 \\
\hline
\end{tabular}

\section{SIMULATION RESULTS}

The proposed harmonic filtering method is applied to demonstrate the damping performances and the effectiveness of the $G-H$ droop control. Figure 2 is a simulation circuit of a Microgrid system operating in the islanding mode, which is rated at $220 \mathrm{~V}$ (line-to-line), $60 \mathrm{~Hz}$. Line impedance is set as $Z_{1}=Z_{2}=0.2+j 0.0754 \Omega$. Parameters of both $\mathrm{DCU}_{1}$ and $\mathrm{DCU}_{2}$ are given in Figure I.

\section{A. $t<1.0 \mathrm{~s}$}

Initially, both $\mathrm{DCU}_{1}$ and $\mathrm{DCU}_{2}$ supply linear loads without any droop controls. Figure 3 shows the power consumption of each DCU is quit unequally distributed.

B. $1.0 \mathrm{~s}<t<1.5 \mathrm{~s}$

After $t=1.0 \mathrm{~s}$, the real power and the reactive power are evenly shared due to the $Q-f$ droop and $P-V$ droop.

\section{C. $1.5 \mathrm{~s}<t<2.0 \mathrm{~s}$}

At $t=1.5 \mathrm{~s}$, all nonlinear loads are switched in, but the harmonic filtering functionality of the DCUs is not yet employed, ie. $G_{1}^{*}=G_{2}^{*}=0$. The sharing of real power and reactive power remains, and the harmonic current of both DCUs results in increased harmonic var as indicated by $H_{1}$ and $H_{2}$. Figure 4(a) shows the line voltages are significantly distorted due to the lack of harmonic filtering. The voltage THDs of bus $\mathrm{A}, \mathrm{B}$, and $\mathrm{C}$ reach $9.2 \%, 9.5 \%$, $9.0 \%$ respectively.

\section{D. $2 \mathrm{~s}<t<2.5 \mathrm{~s}$}

The $G-H$ droop controls of both DCUs are enabled at $t=$ $2 \mathrm{~s}$. Figure 4(b) shows that the voltage THDs of bus A, B, and $\mathrm{C}$ are $1.9 \%, 2.3 \%, 1.5 \%$ respectively, all significantly reduced compared to the results in Figure 4(a). At the steady state, $\mathrm{DCU}_{1}$ absorbs $H_{1}=1892$ var with $G_{1}^{*}=2.17 \Omega^{-1}$ and $\mathrm{DCU}_{2}$ absorbs $H_{2}=1864$ var with $G_{2}^{*}=2.72 \Omega^{-1}$. The dominant harmonic current components of $\mathrm{DCU}_{1}$ and $\mathrm{DCU}_{2}$ are $i_{1}^{5 t h}=3.85 \mathrm{~A}, i_{1}^{7 t h}=2.80 \mathrm{~A}, i_{2}^{5 t h}=3.80 \mathrm{~A}, i_{2}^{7 t h}=$ $2.75 \mathrm{~A}$, as illustrated in Figure 4(e). The harmonic filtering workload is evenly shared between two DCUs thanks to the $G-H$ droop control.

Figure 4(c) shows the waveforms if both $\mathrm{DCU}_{1}$ and $\mathrm{DCU}_{2}$ operate with fixed conductance command, $G_{1}^{*}=G_{2}^{*}=$ $2.5 \Omega^{-1}$. At the steady state, $\mathrm{DCU}_{1}$ consumes 2040 var and $\mathrm{DCU}_{2}$ consumes 1760 var. Figure $4(\mathrm{f})$ shows $i_{1}^{5 t h}=4.09 \mathrm{~A}$, $i_{1}^{7 t h}=3.19 \mathrm{~A}, i_{2}^{5 t h}=3.59 \mathrm{~A}, i_{2}^{7 t h}=2.67 \mathrm{~A}$. Compared to the $G-H$ droop control, the fixed conductance operation results in uneven sharing of harmonic filtering workload, and may cause overloading of certain DCUs.

\section{LABORATORY TEST RESULTS}

Figure 5 shows the laboratory test bench. $\mathrm{DCU}_{1}$ and $\mathrm{DCU}_{2}$ are deployed at bus A and bus B, respectively. The system parameters are given as follows,

- Power system: $110 \mathrm{~V}$ (line-to-line), $60 \mathrm{~Hz}$.

- DCUs: Conventional voltage source inverters with PWM frequency of $20 \mathrm{kHz}$. The droop parameters are as follows, $G_{0}=0 \Omega^{-1}$, 


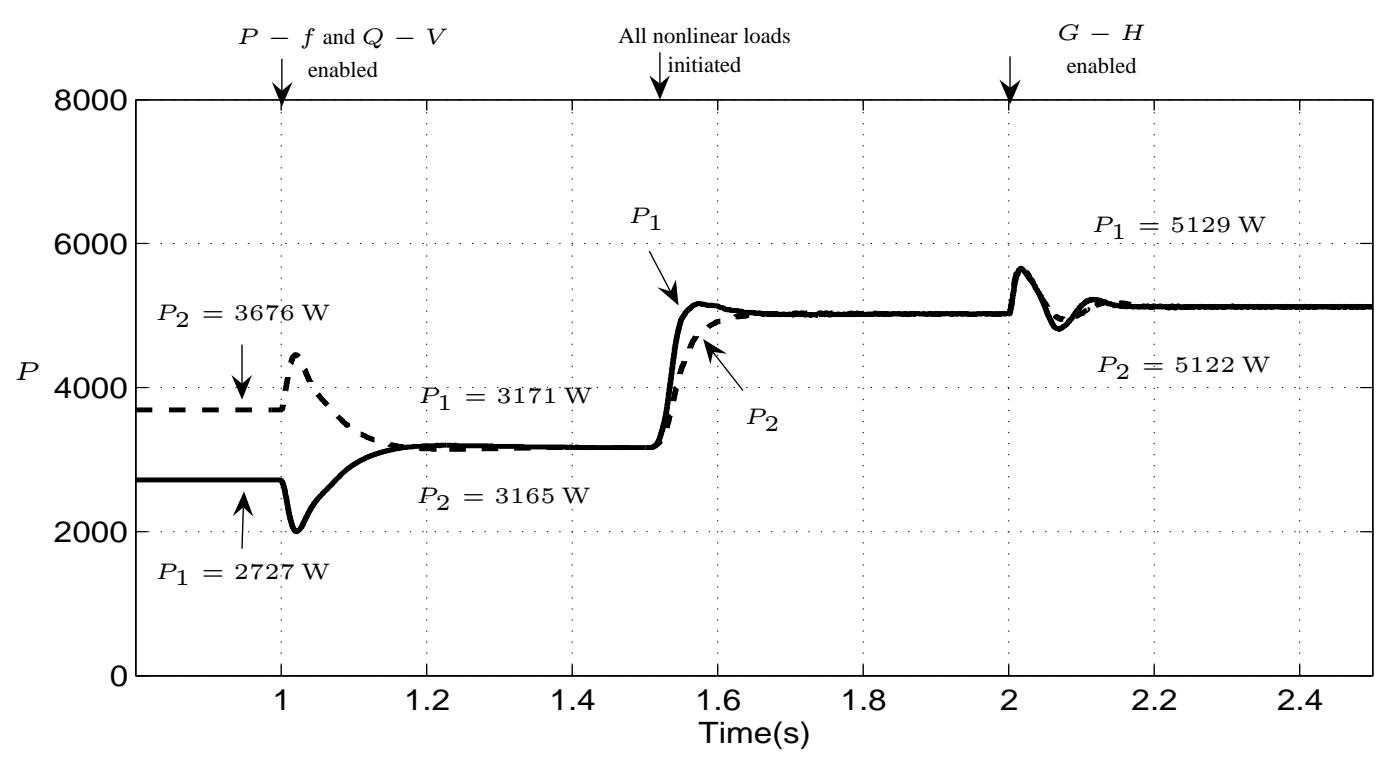

(a) Real power.

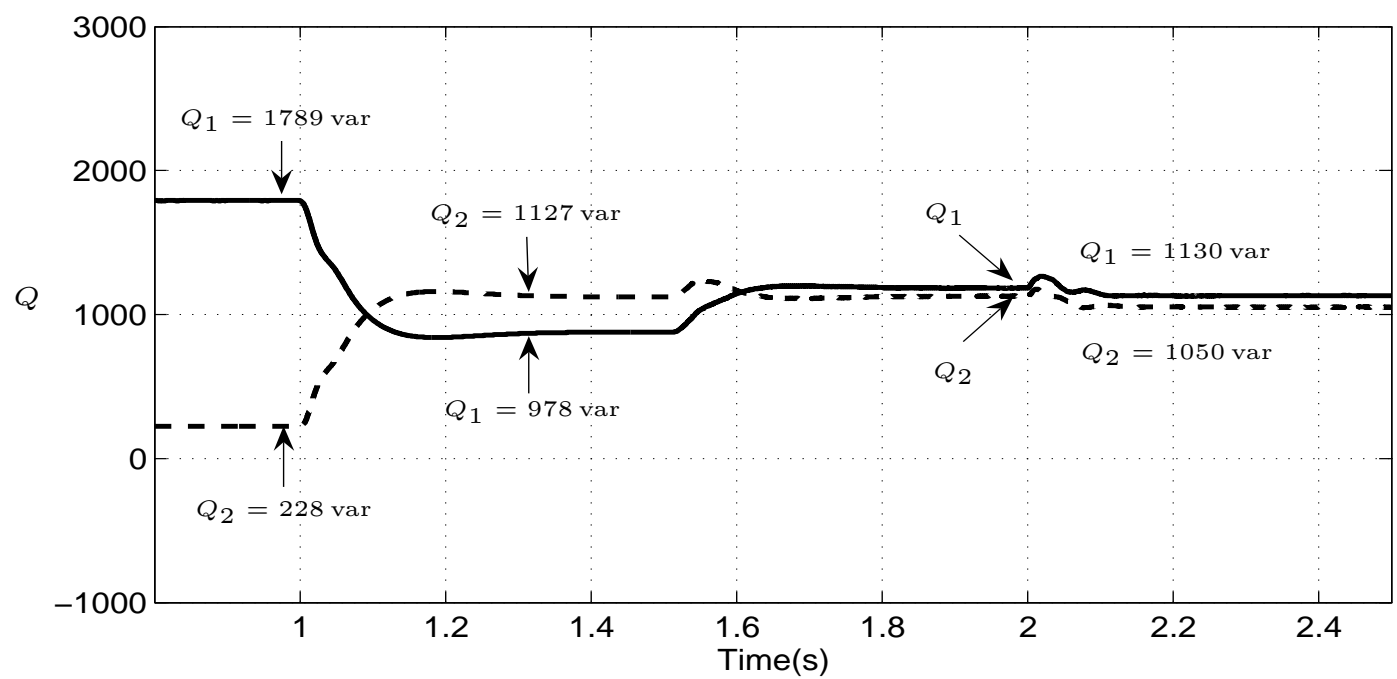

(b) Reactive power.

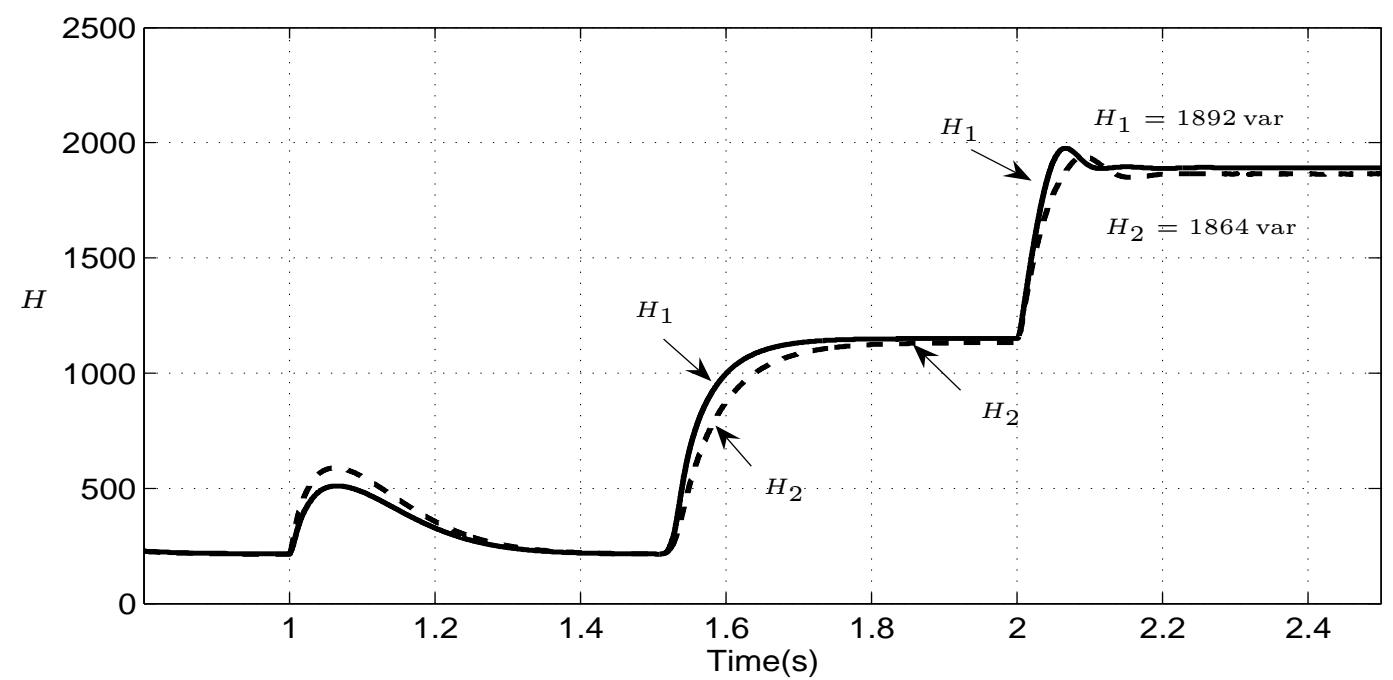

(c) Harmonic var.

Fig. 3. Distributions of the real power, the reactive power, and the harmonic var. (solid line:DCU $\mathrm{D}_{1}$; dash line:DCU $\mathrm{D}_{2}$ ) 


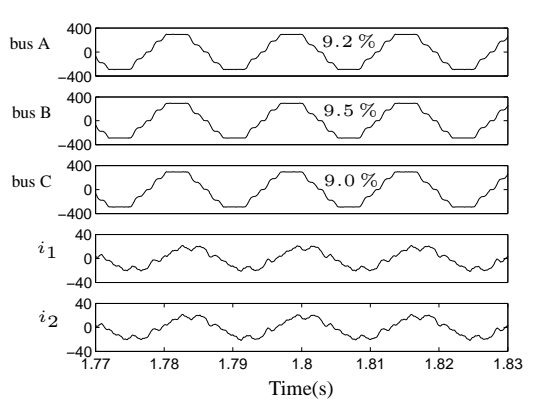

(a) $G_{1}^{*}=G_{2}^{*}=0$.

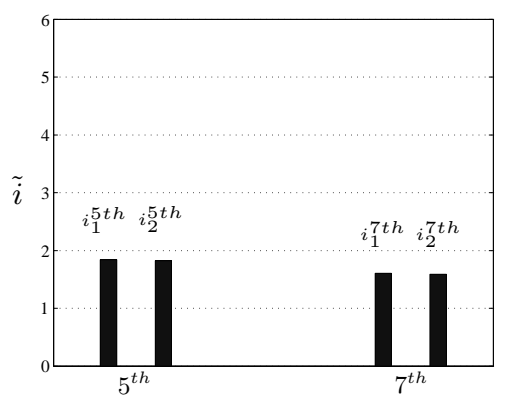

(d) $\tilde{i}$ in Figure 4(a).

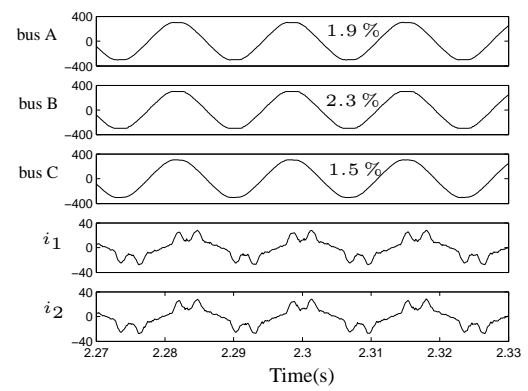

(b) $G-H$ droop is in operation.

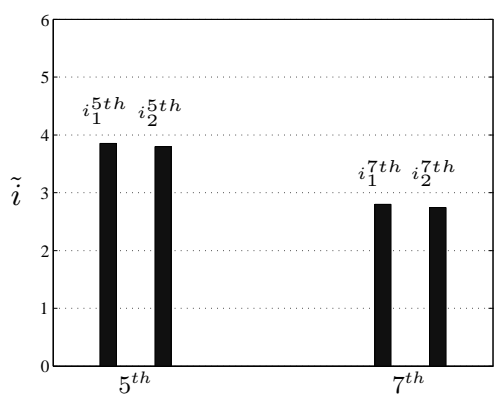

(e) $\tilde{i}$ in Figure 4(b).

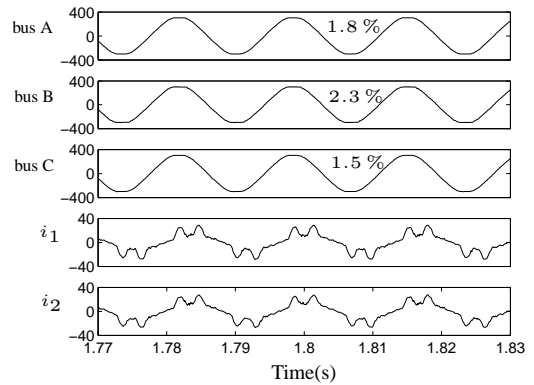

(c) Operate with $G_{1}^{*}=G_{2}^{*}=2.5 \Omega^{-1}$.

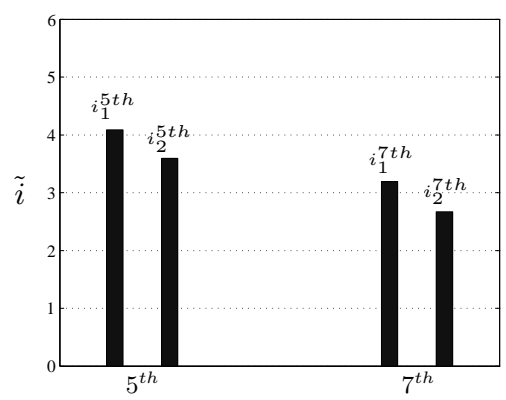

(f) $\tilde{i}$ in Figure 4(c).

Fig. 4. The voltages, currents, fifth harmonic currents, and seventh harmonic currents of both DCUs in different operation modes.

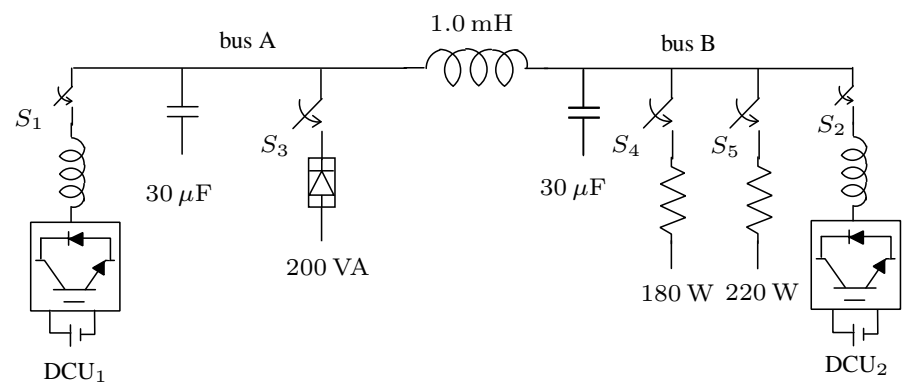

Fig. 5. Laboratory test bench.

$$
\begin{aligned}
& m_{1}=m_{2}=-1 \times 10^{-5} \mathrm{rad} / \mathrm{s} \cdot \mathrm{W}^{-1}, \\
& n_{1}=n_{2}=-1 \times 10^{-3} \mathrm{~A}^{-1}, \\
& b_{1}=b_{2}=-1 \times 10^{-2} \mathrm{~V}^{-2}, \\
& P_{01}=P_{02}=1 \mathrm{~kW}, \\
& Q_{01}=Q_{02}=0 \mathrm{kvar}, \\
& H_{01}=H_{02}=100 \text { var. }
\end{aligned}
$$

Figure 6 shows harmonic var and harmonic conductance commands of both DCUs. When $t<T_{0}$, both DCUs are off. At $t=T_{0}, \mathrm{DCU}_{1}$ is started (both switch $S_{1}$ and switch $S_{5}$ close) and then nonlinear load is turned-on (switch $S_{3}$ closes). $\mathrm{DCU}_{1}$ supplies all the fundamental power and the harmonic filtering var, whereas $\mathrm{DCU}_{2}$ runs under the phase lock mode with zero current command until $t=T_{1}$. At this time interval, $H_{2}$ is due to the distorted line voltage at BUS $\mathrm{B}$ and the current ripple. After $t=T_{1}$, the droop control of $\mathrm{DCU}_{2}$ is engaged, and thus $\mathrm{DCU}_{2}$ gradually increases its power and harmonic var output. At the same time, $G_{1}$ reduces slightly because of the $G-H$ droop control as shown in Figure 6. At the steady state, both DCUs evenly share the real power and harmonic filtering var. The steady-state sharing of harmonic var is given in Figure II(a). When $t=T_{2}$, a newly added linear load (switch $S_{4}$ closes) causes slight decreasing of harmonic distortion. Therefore, the conductance commands of both DCUs are increased to maintain sharing of harmonic var between both DCUs, as given in Figure II(b).

TABLE II

The harmonic conductance command, the harmonic var, the voltage THD, and the real power of both DCUs in Figure 6.

(a) During $T_{1}<t<T_{2}$.

\begin{tabular}{lcccc}
\hline \hline & $\mathrm{G}\left(\Omega^{-1}\right)$ & $\mathrm{H}($ var $)$ & $\mathrm{VTHD}(\%)$ & $\mathrm{P}(\mathrm{W})$ \\
\hline $\mathrm{DCU}_{1}$ & 0.17 & 84 & 3.5 & 200 \\
\hline $\mathrm{DCU}_{2}$ & 0.19 & 81 & 3.1 & 200 \\
\hline
\end{tabular}

(b) During $t>T_{2}$.

\begin{tabular}{ccccc}
\hline \hline & $\mathrm{G}\left(\Omega^{-1}\right)$ & $\mathrm{H}(\mathrm{var})$ & VTHD $(\%)$ & $\mathrm{P}(\mathrm{W})$ \\
\hline $\mathrm{DCU}_{1}$ & 0.30 & 70 & 2.7 & 300 \\
\hline $\mathrm{DCU}_{2}$ & 0.34 & 66 & 2.4 & 300 \\
\hline
\end{tabular}

\section{DISCUSSION}

The G-H droop control manipulates the harmonic conductance of each DCU to achieve the proportional sharing of the total harmonic reactive power of the load. 


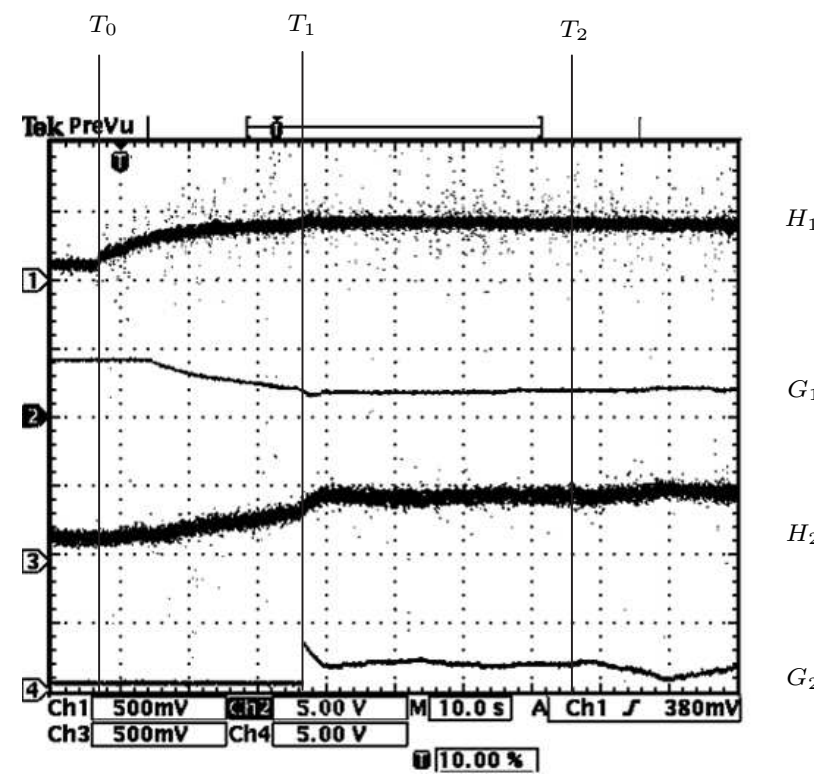

Fig. 6. Time response of the harmonic var, the harmonic conductance command, and the real power for both DCUs with the same rated capacity, $H_{1}, H_{2}: 100 \mathrm{var} / d i v, \quad G_{1}, G_{2}:^{0.1 \Omega^{-1} / V}, \mathrm{X}$ axis: 10 s/div.

However, the power line impedance could also affect the flow of harmonic current. Also, in the low voltage Microgrid, the line tends to have higher resistive content than the conventional transmission/distribution network as previous literatures have pointed out $[17,18]$. The performance of the $\mathrm{G}-\mathrm{H}$ droop control is examined under these circumstances using a simplified circuit as shown in Figure 7.

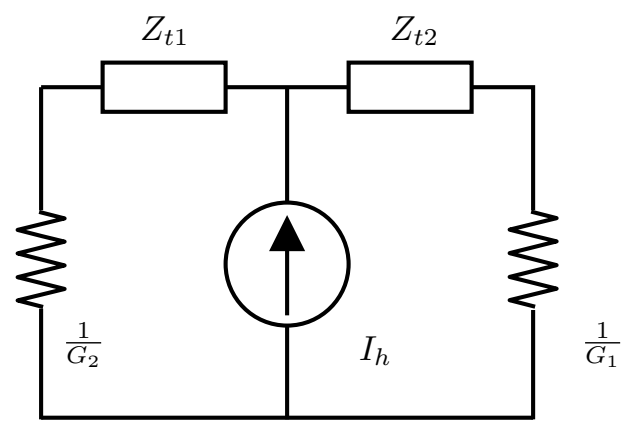

Fig. 7. Simplified one-line diagram of the harmonic producing load and the DCUs' harmonic conductances.

The harmonic current source $I_{h}$ represents the harmonic current of a $1.0 \mathrm{pu}$. nonlinear load with $0.2 \mathrm{pu} ., 0.15 \mathrm{pu}$., $0.9 \mathrm{pu}$. and $0.77 \mathrm{pu}$. of the $5^{\text {th }}, 7^{\text {th }}, 11^{\text {th }}$ and $13^{\text {th }}$ harmonic current respectively.

The harmonic current and the harmonic reactive power absorbed by each DCUs can be represented as

$$
\begin{array}{r}
I_{1}=\frac{\frac{1}{G_{2}}+Z_{t 2}}{\frac{1}{G_{1}}+\frac{1}{G_{2}}+Z_{t 1}+Z_{t 2}} \\
I_{2}=\frac{\frac{1}{G_{1}}+Z_{t 1}}{\frac{1}{G_{1}}+\frac{1}{G_{2}}+Z_{t 1}+Z_{t 2}} \\
H_{1}=E_{r m s} \cdot G_{1} \cdot I_{1, r m s} \\
H_{2}=E_{r m s} \cdot G_{2} \cdot I_{2, r m s}
\end{array}
$$

The line impedance $Z_{2}$ is assumed to be $0.075 \Omega$, $0.075 \angle\left(\frac{\pi}{4}\right) \Omega$, and $0.075 \angle\left(\frac{\pi}{2}\right) \Omega$, and $Z_{1}$ varies from 0 to $0.15 \Omega$ at the same impedance angle of $Z_{2}$. In order to verify if the G-H droop control can accomplish even sharing of the harmonic reactive power, the ratio of $\frac{H_{1}}{H_{2}}$ and their $G_{1}$ and $G_{2}$ commands are plotted in Figure 8 and Figure 9 for the droop slope of $-2 \times 10^{-2} \mathrm{~V}^{-1}$ and $-5 \times 10^{-3} \mathrm{~V}^{-1}$ respectively. The G-H droop settings of both DCUs are identical, thus the harmonic VARs should split evenly between them.

Figure 8 shows that the harmonic reactive power sharing of G-H droop is affected by the varying line impedance $Z_{1}$. For the mismatch of $-30 \%$ and $+30 \%$ of the line impedances $Z_{1}$ and $Z_{2}$, the ratio $H_{1} / H_{2}$ deviates in the range of $+47 \% \sim-24 \%$ in the case of inductive line, and $+15 \% \sim-12 \%$ in the case of resistive line. The G-H droop is more effective in the case of resistive line because the active conductance commands $G_{1}$ and $G_{2}$ of both DCUs are also resistive, they have direct impact on the current division as given in Figure 12. For the inductive line, the active conductance commands affect the current division through a quadratic relationship with the reactance, and the G-H droop is not as effective in terms of the sharing of harmonic VARs. Also, for higher order harmonics, the line impedance grows proportionally with the harmonic frequency, while the resistive line impedance remains the same across the spectrum. Thus the conductance commands become less effective for the high order harmonics. This can be improved if separate conductance commands are adopted for different harmonic frequencies [19]. The comparison between Figure 8 and Figure 9 also shows that the steeper droop slope results in more balanced sharing when the line impedance $\left|Z_{1}\right|$ is small. As $\left|Z_{1}\right|$ increases, the line impedance dominates the current division, and the active conductance commands become less effective in balancing the harmonic VARs.

\section{SUMMARY}

An autonomous harmonic filtering strategy is proposed for distributed energy resource converters in the Microgrid. The harmonic conductance-harmonic var $(G-H)$ droop control is developed to accomplish balanced distribution of harmonic filtering workload among multiple DCU converters. With proper settings of the $P-f, Q-V$, and $G-H$ droop controls as given in Figure 3,Figure 4, Figure 5 and Figure 7, each unit can share the real power, the reactive power and the harmonic filtering workload based on its own rated capacity without any communications. The $P-f$ and $Q-V$ droop methods have been widely accepted for the distributed controls of power converters. The proposed $G-H$ droop 


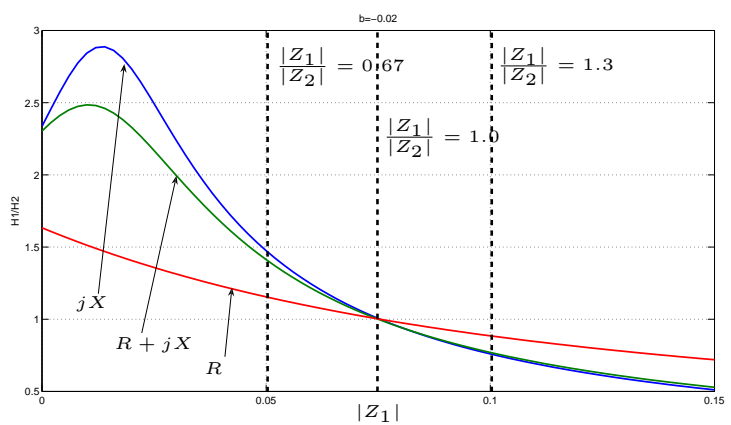

(a) Sharing of harmonic reactive power $H_{1}$ and $H_{2}$.

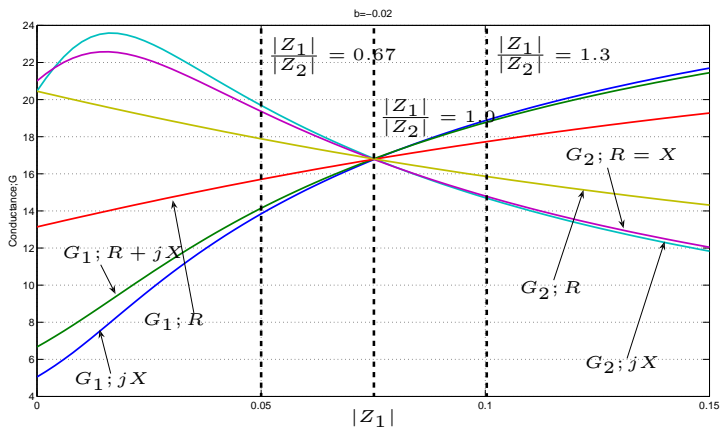

(b) Harmonic conductance commands $G_{1}$ and $G_{2}$.

Fig. 8. Effect of line impedances on the G-H droop; droop slope $b=-2.0 \times 10^{-2} V^{-3}$.

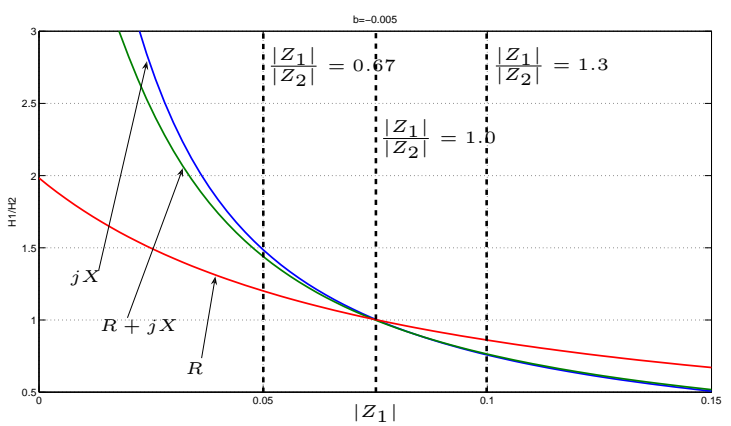

(a) Sharing of harmonic reactive power $H_{1}$ and $H_{2}$.

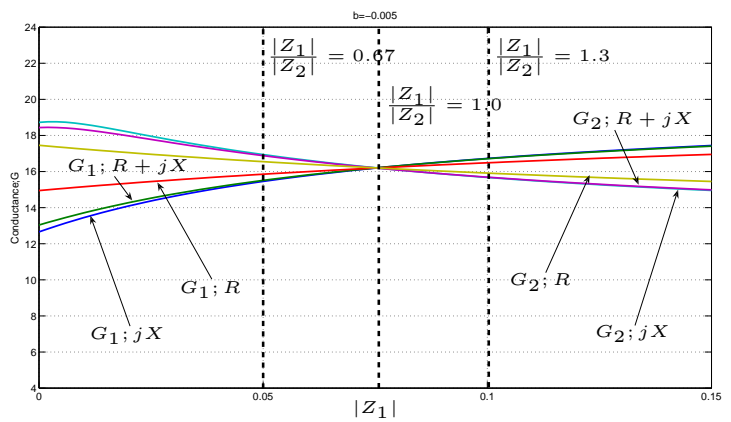

(b) Harmonic conductance commands $G_{1}$ and $G_{2}$.

Fig. 9. Effect of line impedances on the G-H droop; droop slope $b=-5.0 \times 10^{-3} V^{-1}$. control can be seamlessly integrated with $P-f$ and $Q-V$ droop controls to provide harmonic voltage damping within an islanding network. The $G-H$ droop control can also reduce the harmonic voltage distortion in the grid-connected operation $[20,21]$. The impact of the line impedance on the $G-H$ droop control is also investigated. The $G-H$ droop characteristics need to be tuned to ensure that the active harmonic conductance commands of DCUs are sufficiently significant compared to the line impedance. This could be done by vertically shifting the droop lines or adjusting the rated capacities of DCUs [3,22]. In general, the $G-H$ droop control is more effective if the line is resistive as shown in the previous analysis. Thus it is suitable for the low-voltage Microgrid applications.

\section{REFERENCES}

[1] R. Lasseter. Microgrids. In Power Engineering Society Winter Meeting, pages 305-308, 2002.

[2] R. Lasseter and P. Piagi. Providing premium power through distributed resources. In Proc. 33rd Hawaii Int. Conf. System Sciences, pages 1437-1445, 2002.

[3] M. C. Chandorkar, D. M. Divan, and R. Adapa. Control of parallel connected inverters in standalone AC supply systems. IEEE Trans. Ind. Applicat., 29(1):136-143, Jan./Feb. 1993.

[4] E. A. A. Coelho, P. C. Cortizo, and P. F. D. Garcia. Smallsignal stability for parallel-connected inverters in a standalone AC supply system. IEEE Trans. Ind. Applicat., 38(2):533-542, Mar/Apr. 2002.

[5] Y. Li, D. M. Vilathgamuwa, and P. C. Loh. Design, analysis, and real-time testing of a controller for multibus microgrid system. IEEE Trans. Power Electron., 19(5):1195-1204, Sept. 2004.

[6] J. M. Guerrero, L. G. de Vicuna, J. Matas, M. Castilla, and J. Miret. A wireless controller to enhance dynamic performance of parallel inverters in distributed generation systems. IEEE Trans. Power Electron., 19(5):1205-1213, Sept. 2004.

[7] J. H. R. Enslin and P. J. M. Heskes. Harmonic interaction between a large number of distributed power inverters and the distribution network. IEEE Trans. Power Electron., 19(6):1586-1593, Nov. 2004.

[8] H. Akagi, H. Fujita, and K. Wada. A shunt active filter based on voltage detection for harmonic termination of a radial power distribution line. IEEE Trans. Ind. Applicat., pages 638-645, May/Jun. 1999.

[9] V. Blasko and V. Kaura. A novel control to actively damp resonance in input LC filter of a three-phase voltage source converter. IEEE Trans. Ind. Applicat., 33(2):542550, Mar./Apr. 1997.

[10] U. Borup, P. N. Enjeti, and F. Blaabjerg. A new spacevector-based control method for UPS systems powering nonlinear and unbalanced loads. IEEE Trans. Ind. Applicat., 37(6):1864-1870, Nov./Dec. 2001.

[11] T.. Takeshita and N. Matsui. Current waveform control of pwm converter system for harmonic suppression on distribution system. IEEE Trans. Ind. Electron., 50(6):1134-1139, Dec. 2003. 
[12] A. Tuladhar, H. Jin, T. Unger, and K. Mauch. Control of parallel inverters in distributed AC power systems with consideration of line impedance effect. IEEE Trans. Ind. Applicat., 36(1):131-137, Jan./Feb. 2000.

[13] U. Borup, F. Blaabjerg, and P. N. Enjeti. Sharing of nonlinear load in parallel-connected three-phase converters. IEEE Trans. Ind. Applicat., 37(6):1817-1823, Nov./Dec. 2001.

[14] T.-L. Lee and P.-T. Cheng. Design of a new cooperative harmonic filtering strategy for distributed generation interface converters in an islanding network. IEEE Trans. Power Electron., 42(5):1301-1309, Sept. 2007.

[15] H. Akagi, Y. Kanagawa, and A. Nabase. Instantaneous reactive power compensator comprising switching devices without energy storage components. IEEE Trans. Ind. Applicat., IA-20, May/Jun. 1984.

[16] E. H. Watanabe, R. M. Stephan, and M. Aredes. New concepts of instantaneous active and reactive powers in electrical systems with generic loads. IEEE Trans. Power Delivery, 8(2):697-703, Apr. 1993.

[17] Karel De Brabandere, Bruno Bolsens, Jeroen Van den Keybus, Achim Woyte, Johan Driesen, and Ronnie Belmans. A voltage and frequency droop control method for parallel inverters. Power Electronics, IEEE Transactions on, 22(4):1107-1115, July 2007.

[18] A. Engler and N. Soultanis. Droop control in LV-grids. In Future Power Systems, 2005 International Conference on, pages 6 pp.-6, 2005.

[19] Tzung-Lin Lee, Jian-Cheng Li, and Po-Tai Cheng. Discrete frequency tuning active filter for power system harmonics. IEEE Trans. Power Electron., 24(5):12091217, May 2009.

[20] P. T. Cheng and T. L. Lee. Distributed active filter systems (DAFSs): A new approach to power system harmonics. IEEE Trans. Ind. Applicat., 42(5):13011309, Sept./Oct. 2006.

[21] P. T. Cheng and T. L. Lee. Analysis of harmonic damping effect of the distributed active filter system. IEEJ Trans.
Ind. Applicat., 126(5):605-614, May 2006.

[22] T.-L. Lee, P.-T. Cheng, H. Akagi, and H. Fujita. A dynamic tuning method for distributed active filter systems. IEEE Trans. Ind. Appl., 44(2):612-623, Mar./Apr. 2008.

\section{BIOGRAPHIES}

Tzung-Lin Lee received the B.S. degree in electrical engineering from Chung Yuan Christian University, Taoyuan, TAIWAN, in 1993, the M.S. degree in electrical engineering from National Chung Cheng University, Chiayi, TAIWAN, in 1995, and the $\mathrm{Ph} . \mathrm{D}$. degree in electrical engineering from National Tsing Hua University, Hsinchu, TAIWAN, in 2007. From 1997 to 2001, he was a research engineer at the Microwave Department in Electronics Research \& Service Organization (ERSO), Industrial Technology Research Institute (ITRI), Hsinchu, TAIWAN. He is currently an Assistant Professor in the department of electrical engineering, National Sun Yat-sen University, Kaohsiung, TAIWAN. His research interests are in utility applications of power electronics.

Chia-Tse Lee received the B.S. degree in electrical engineering from National Tsing Hua University, Hsinchu, Taiwan in 2007. He is currently pursuing the Ph.D. degree in the same university. His research interests include power electronics for distributed generation systems and power converter controls.

Po-Tai Cheng received the B.S. degree from National Chiao Tung University, Hsinchu, Taiwan in 1990 and M.S.E.E. and Ph.D. degrees from the University of Wisconsin, Madison, WI, USA in 1994 and 1999 respectively. He is currently a professor in the Department of Electrical Engineering, National Tsing Hua University, Hsinchu, Taiwan. His research interests include distributed generation systems, utility applications of power electronics, power quality issues and high power converters. 